\title{
Useful Inequalities for Upper and Lower Bounds for the Repairable Aging Cold Standby System
}

\author{
Gökhan GÖKDERE* \\ Fırat University Faculty of Science, Department of Statistics, Elazığ, Turkey
}

\begin{abstract}
In this paper, we study a cold standby repairable system consisting of two non-identical components and a single repair facility with one repairman. It is assumed that, one of the two components is operating while the other is in cold standby and also two components follow a geometric process. Under these assumptions, at first we present LaplaceStieltjes transform of the system lifetime and then we give some useful inequalities about the mean lifetime of the system.
\end{abstract}

\section{Introduction}

Cold standby redundancy technique uses one or more redundant components that are unpowered, do not consume any energy and do not fail until being activated to replace a faulty online component. Whenever working component fails, then an available cold standby component, i.e. inactive standby component, is immediately powered up to take over the mission task. Some recent works on the research of the cold standby systems are in [1-3].

In practice after the repair, most repairable components are not "as good as new". To pay attention this problem, much work has been done by [4-7]. For an imperfect repair model, it is more acceptable to consider that the successive operating times of the component after repair will be even shorter while the consecutive repair times of the component after fail will be even longer [8-11].

Consider a redundant system consisting of two non-identical components called component 1 and component 2 and a single repair facility with one repairman. Initially, one component begins to operate, the other component is inactive standby. As soon as the operating component fails, the standby component is switched on immediately and failed component is taken for repair. When repair of one component is completed and the other component is still operating the repaired component returns to the standby pool and is once again available to be used as operating component. The system is breakdown when the working component fails while the other component is under repair.

\footnotetext{
* Corresponding author: g.g.gokdere@gmail.com
} 


\section{Lifetime of the system}

In this section, at first we will investigate possible malfunction situations of the system and then we will define the system lifetime measured from the moment when component 1 fails.

We make the following assumptions concerning failures and repairs.

Assumption 1. Failure time of component $k$ is the random variable $X_{k}^{(n)}$ with the distribution function

$$
F_{X_{k}}^{(n)}(t)=F_{k}\left(m_{k} t\right), k=1,2 ; t \geq 0,
$$

where $m_{k}=\left\{\begin{array}{ll}a^{n}, & k=1 \\ a^{n-1}, & k=2\end{array}\right.$ and $a>1$ is the ratio of the geometric process (GP) for component $k$.

Assumption 2. Repair time of component $k$ is the random variable $Y_{k}^{(n)}$ with the distribution function

$$
G_{Y_{k}}^{(n)}(t)=G_{k}\left(b^{n-1} t\right), k=1,2 ; t \geq 0,
$$

where $0<b<1$ is the ratio of the GP for component $k$.

Assumption 3. $X_{k}{ }^{(1)}, X_{k}{ }^{(2)}, \ldots$ be successive working times and $Y_{k}{ }^{(1)}, Y_{k}{ }^{(2)}, \ldots$ be successive repair times of component $k$. It is supposed that all these random variables are independent.

Let $\tau_{12}(a, b)$ denote the system lifetime under the assumption that at initial instant $t=0$, component 1 begins to work and component 2 is inactive standby. Let $\tau(a, b)$ denote the system lifetime measured from the moment when component 1 fails. It is evident that $\tau_{12}(a, b)=X_{1}+\tau(a, b)$, where $X_{1}$ and $\tau(a, b)$ are independent random variables.

The system described above breakdown when for some $n$ either event

$A_{n}=\left\{X_{2}^{(j)}>Y_{1}^{(j)}, X_{1}^{(j)}>Y_{2}^{(j)}, j=1,2, \ldots, n-1 ; \hat{X}_{2}^{(n)} \leq Y_{1}^{(n)}\right\}$

or event

$B_{n}=\left\{X_{2}^{(j)}>Y_{1}^{(j)}, X_{1}^{(j)}>Y_{2}^{(j)}, j=1,2, \ldots, n-1 ; X_{2}^{(n)}>Y_{1}^{(n)}, \hat{X}_{1}^{(n)} \leq Y_{2}^{(n)}\right\}$

occurs. Note that $\alpha_{12}(n)$ is the probability that after the $n$-th repair working component 2 does not fail while component 1 is under $(n+1)$-th repair and $\alpha_{21}(n)$ is the probability that after the $(n+1)$-th repair, working component 1 does not fail while component 2 is under $n$-th repair. Then $\alpha_{12}(n)$ and $\alpha_{21}(n)$ can be represented as for $i=1,2$ and $i \neq k$

$$
\alpha_{i k}(n)=P\left\{X_{k}^{(n)}>Y_{i}^{(n)}\right\}=\int_{0}^{\infty} G_{Y_{i}}^{(n)}(t) d F_{X_{k}}^{(n)}(t)
$$

Then, the probability that the system breakdown is obtained as

$$
\alpha(n)=1-\alpha_{12}(n) \alpha_{21}(n) .
$$

Thus using (1) and (2), the probabilities of events $A_{n}$ and $B_{n}$ are found to be

$$
P\left\{A_{n}\right\}=\prod_{j=1}^{n-1}(1-\alpha(j)) \bar{\alpha}_{12}(n) \quad \text { and } \quad P\left\{B_{n}\right\}=\prod_{j=1}^{n-1}(1-\alpha(j)) \alpha_{12}(n) \bar{\alpha}_{21}(n)
$$

where $\bar{\alpha}_{12}(n)=1-\alpha_{12}(n), \bar{\alpha}_{21}(n)=1-\alpha_{21}(n)$ and $n \geq 1$. 
Then the lifetime of the system is represented as

$$
\begin{array}{r}
P\left\{\tau(a, b)=\sum_{j=1}^{n-1} \zeta_{j}+\hat{X}_{2}^{(n)}\right\}=\prod_{j=1}^{n-1}(1-\alpha(j)) \bar{\alpha}_{12}(n), \\
P\left\{\tau(a, b)=\sum_{j=1}^{n-1} \zeta_{j}+X_{2}^{(n)}+\hat{X}_{1}^{(n)}\right\}=\prod_{j=1}^{n-1}(1-\alpha(j)) \alpha_{12}(n) \bar{\alpha}_{21}(n)
\end{array}
$$

where $\zeta_{j}=X_{2}^{(j)}+X_{1}^{(j)}$ and for $n=1$ the sum and product vanishes.

\section{Laplace-Stieltjes transform of the system lifetime}

In this section, our objective is to find Laplace-Stieltjes (LS) transform of the system lifetime $\tau_{12}(a, b)$ with the help of the $\tau(a, b)$ which is defined previous section. We now introduce the following notations;

$$
\begin{aligned}
& q_{i k(p)}(s)=\int_{0}^{\infty} e^{-s t} G_{Y_{i}}^{(n)}(t) d F_{X_{k}}^{(n)}(t) \\
& \bar{q}_{i k(p)}(s)=\int_{0}^{\infty} e^{-s t} \bar{G}_{Y_{i}}^{(n)}(t) d F_{X_{k}}^{(n)}(t)=f_{X_{k}}^{(n)}(s)-q_{i k(p)}(s) ; \\
& f_{X_{k}}^{(n)}(s)=\int_{0}^{\infty} e^{-s t} d F_{X_{k}}^{(n)}(t)
\end{aligned}
$$

where $i=1,2 ; k=1,2 ; i \neq k$ and $\bar{G}_{Y_{i}}^{(n)}(t)=1-G_{Y_{i}}^{(n)}(t)$. Using the formula of total expectation and (3)-(4) we have

$$
\begin{aligned}
E e^{-s \tau(a, b)}= & \sum_{n=1}^{\infty}\left(E e^{-s\left(\zeta_{1}+\cdots+\zeta_{n-1}+\hat{X}_{2}^{(n)}\right)} \prod_{j=1}^{n-1}(1-\alpha(j)) \bar{\alpha}_{12}(n)\right. \\
& \left.+E e^{-s\left(\zeta_{1}+\cdots+\zeta_{n-1}+X_{2}^{(n)}+\hat{X}_{1}^{(n)}\right)} \prod_{j=1}^{n-1}(1-\alpha(j)) \alpha_{12}(n) \bar{\alpha}_{21}(n)\right)
\end{aligned}
$$

Clearly, using (1) and (5)-(7), following equations can be obtained as

$$
\begin{gathered}
E e^{-s X_{k}^{(j)}}=\int_{0}^{\infty} e^{-s t} d P\left\{X_{k}^{(j)}<t \mid X_{k}^{(j)}>Y_{i}^{(j)}\right\}=\frac{q_{i k(j)}(s)}{\alpha_{i k}(j)} \\
E e^{-s \hat{X}_{k}^{(n)}}=\int_{0}^{\infty} e^{-s t} d P\left\{X_{k}^{(n)}<t \mid X_{k}^{(n)}<Y_{i}^{(n)}\right\}=\frac{\bar{q}_{i k(n)}(s)}{\bar{\alpha}_{i k}(n)}
\end{gathered}
$$

The proof of (9) and (10) are presented in [12]. 
Taking into consideration Assumption 3 and substituting (9)-(10) in (8) we find that

$$
E e^{-s \tau(a, b)}=\sum_{n=1}^{\infty}\left(\bar{q}_{12(n)}(s)+q_{12(n)}(s) \bar{q}_{21(n)}(s)\right) \prod_{j=1}^{n-1}\left(q_{12(j)}(s) q_{21(j)}(s)\right) .
$$

In view of $\tau_{12}(a, b)=X_{1}+\tau(a, b)$ and $f_{X_{k}}(t)=E e^{-s X_{k}}=\int_{0}^{\infty} e^{-s t} d F_{X_{k}}(t), k=1,2$ the LS transform of the system lifetime $\tau_{12}(a, b)$ is obtained as

$$
\begin{aligned}
& E e^{-s \tau_{12}(a, b)=f_{X_{1}}(s)} \\
& \qquad \times\left(\sum_{n=1}^{\infty}\left(\bar{q}_{12(n)}(s)+q_{12(n)}(s) \bar{q}_{21(n)}(s)\right) \prod_{j=1}^{n-1}\left(q_{12(j)}(s) q_{21(j)}(s)\right)\right) .
\end{aligned}
$$

The LS transform of the system lifetime given by the equation (11) is required to calculate the expected value of the system's lifetime, i.e, the average life expectancy. However, calculation of this transform is not always possible. Hence, it is very important to determine the lower and upper bounds for the LS transform of the system lifetime and depending on this the average life expectancy of the system. In GP model, because of the machine operating time will be reduced gradually after each repair, we can write the following result.

Remark 1. For mean lifetime of the system, we have

$$
E\left\{\tau_{12}(a, b)\right\} \leq E\left\{\tau_{12}(1,1)\right\} .
$$

Remark 2. From (11) and GP model, we have

$$
E e^{-s \tau_{12}(a, b)} \geq E e^{-s \tau_{12}(1,1)} .
$$

\section{Analyzing the lifetime of the system when the failure time is fixed}

Let failure time of component $k$ is fixed and failure repair for component $k$ follows GP repair. Then, the LS transform of the system lifetime is given by the following theorem.

Theorem 1. Let the failure time of component $\mathrm{k}$ is the random variable $\mathrm{X}_{\mathrm{k}}$ with the distribution function

$$
F_{X_{k}}(t)=F_{k}\left(m_{k} t\right)= \begin{cases}1 & m_{k} t \geq c \\ 0 & m_{k} t<c\end{cases}
$$

where $k=1,2 ; t \geq 0 ; c>0 ; m_{k}=\left\{\begin{array}{ll}a^{n}, & k=1 \\ a^{n-1}, & k=2\end{array}\right.$ and failure repair for component $k$ holds Assumption 2.2. Then

$$
\begin{aligned}
E e^{-s \tau_{12}(a, b)}= & e^{-s c}\left(\sum _ { n = 1 } ^ { \infty } \left(e^{-s c / a^{n-1}}\left(1-G_{1}\left(\frac{b^{n-1} c}{a^{n-1}}\right)\right)+e^{-s c(a+1) / a^{n}} G_{1}\left(\frac{b^{n-1} c}{a^{n-1}}\right)\right.\right. \\
& \left.\times\left(1-G_{2}\left(\frac{b^{n-1} c}{a^{n}}\right)\right) \prod_{j=1}^{n-1} e^{-s c(a+1) / a^{j}} G_{1}\left(\frac{b^{j-1} c}{a^{j-1}}\right) G_{2}\left(\frac{b^{j-1} c}{a^{j}}\right)\right) .
\end{aligned}
$$


Proof. Substituting the expressions (12) in (5)-(7) and also considering Assumption 2.2, we obtain

$$
\begin{gathered}
q_{12(p)}(s)=\int_{0}^{\infty} e^{-s t} G_{Y_{1}}^{(p)}(t) d F_{X_{2}}^{(p)}(t)=e^{-s c / a^{p-1}} G_{1}\left(\frac{b^{p-1}}{a^{p-1}} c\right), \\
q_{21(p)}(s)=\int_{0}^{\infty} e^{-s t} G_{Y_{2}}^{(p)}(t) d F_{X_{1}}^{(p)}(t)=e^{-s c / a^{p}} G_{2}\left(\frac{b^{p-1}}{a^{p}} c\right), \\
\bar{q}_{12(p)}(s)=\int_{0}^{\infty} e^{-s t} \bar{G}_{Y_{1}}^{(p)}(t) d F_{X_{2}}^{(p)}(t)=e^{-s c}-q_{12(p)}(s), \\
\bar{q}_{21(p)}(s)=\int_{0}^{\infty} e^{-s t} \bar{G}_{Y_{2}}^{(p)}(t) d F_{X_{1}}^{(p)}(t)=e^{-s c}-q_{21(p)}(s), \\
f_{X_{1}}^{(p)}(s)=e^{-s c / a^{p}}, f_{X_{2}}^{(p)}(s)=e^{-s c / a^{p-1}}, f_{X_{k}}(s)=e^{-s c} .
\end{gathered}
$$

Now substituting above expressions in (11) and then making suitable simplifications, we get the proof.

Theorem 2. Mean time to failure of the system for $a=1$ and $b=1$, we have

$$
E\left\{\tau_{12}(1,1)\right\}=c+\frac{c\left(1+G_{1}(c)\right)}{1-G_{1}(c) G_{2}(c)} .
$$

Proof. Let $a=1$ and $\mathrm{b}=1$ in (13), then

$$
E e^{-s \tau_{12}(1,1)}=\frac{e^{-2 s c}\left(1-G_{1}(c)\right)+e^{-3 s c} G_{1}(c)\left(1-G_{2}(c)\right)}{1-e^{-2 s c} G_{1}(c) G_{2}(c)} .
$$

From $(15)$ and $E \tau_{12}(1,1)=-\frac{d}{d s} E e^{-s \tau_{12}(1,1)}(0)$ we can get the proof.

Remark 3. From Remark 2, we have

$$
E e^{-s \tau_{12}(a, b)} \geq \frac{e^{-2 s c}\left(1-G_{1}(c)\right)+e^{-3 s c} G_{1}(c)\left(1-G_{2}(c)\right)}{1-e^{-2 s c} G_{1}(c) G_{2}(c)} .
$$

Remark 4. For mean lifetime of the system, we have

$$
2 c \leq E\left\{\tau_{12}(a, b)\right\} \leq \frac{2 c+c G_{1}(c)-c G_{1}(c) G_{2}(c)}{1-G_{1}(c) G_{2}(c)} .
$$

Remark 4 is very important in terms of giving the upper and lower bounds of the average operating time of the system under the restrictions of fixed operating time and repair time which is defined depending on GP. Now let us examine the following exception for the mean lifetime of the system. Let failure repair for component $k$ be exponential, then the following theorem holds for the upper bound of the mean lifetime of the system. 
Theorem 3. Let failure time of component $k$ is fixed as described in (12) and failure repair for component $k$ be exponential with cumulative distribution function

$$
G_{Y_{k}}(t)=1-e^{-\mu_{k} t},
$$

where $t \geq 0$ and $\mu_{k} \geq 0 ; k=1,2$. Then, under the restriction $\mu_{2} \geq \mu_{1}$ and $\mu_{1}+\mu_{2}=u(u$ is constant),

$$
E\left\{\tau_{12}(1,1)\right\} \leq c+\frac{c}{e^{-\frac{u c}{2}}}
$$

Proof. According (16), (14) takes the form

$$
E\left\{\tau_{12}(1,1)\right\}=c+\frac{c+c\left(1-e^{-\mu_{1} c}\right)}{e^{-\mu_{1} c}+e^{-\mu_{2} c}-e^{-\left(\mu_{1}+\mu_{2}\right) c}} .
$$

From $\mu_{2} \geq \mu_{1}$ and $\mu_{1}+\mu_{2}=u$ it follows that

$$
\begin{aligned}
E\left\{\tau_{12}(1,1)\right\} & \leq c+\frac{2 c-c e^{-\mu_{1} c}}{2 e^{-\frac{u c}{2}}-e^{-\mu_{1} c} e^{-\mu_{2} c}} \\
& \leq c+\frac{2 c-c e^{-\mu_{1} c}}{2 e^{-\frac{u c}{2}}-e^{-\mu_{1} c} e^{-\frac{u c}{2} c}} .
\end{aligned}
$$

From this we get the proof.

\section{Conclusions}

In this article, we have studied a repairable aging cold standby system with a single standby unit when the components are independent. We have presented LS transform of the system lifetime and compute its expected value when the failure time is fixed. Finally, we aim to construct upper and lower bounds belongs to the system reliability by finding some inequalities corresponding to reliability of the technical system. The results of the study are important and can be applicable to a cold standby repairable system with preventive repair.

\section{References}

1. G. Levitin, L. Xing and Y. Dai, Cold-standby sequencing optimization considering mission cost, Reliability Engineering and System Safety, 118, 28-34(2013).

2. S. Eryllmaz, A study on reliability of coherent systems equipped with a cold standby component, Metrika, 77, 349-359(2014).

3. Q. Wu and $\mathrm{S}$. Wu, Reliability analysis of two-unit cold standby repairable systems under Poisson shocks, Applied Mathematics and Computation, 218, 171-182(2011).

4. M. Brown and F. Proschan, Imperfect repair, Journal of Applied Probability, 20, 851859(1983).

5. K.S. Park, Optimal number of minimal repairs before replacement, IEEE Transactions on Reliability, 28, 137-140(1979).

6. M. Kijima, Some results for repairable system with general repair, Journal of Applied Probability, 26, 89-102(1989). 
7. V. Makis and A.K.S. Jardine, A note on optimal replacement policy under general repair, European Journal of Operational Research, 69, 75-82(1993).

8. Y. Lam, Geometric processes and replacement problem, Acta Mathematicae Applicatae Sinica, 4, 366-377(1988).

9. Y. Lam, A note on the optimal replacement problem, Advances in Applied Probability, 20, 479-482(1988).

10. Y. L. Zhang, An optimal geometric process model for a cold standby repairable system, Reliability Engineering and System Safety, 63, 107-110(1999).

11. Y.L. Zhang, A geometric process repair model for a repairable system with delayed repair, Computers and Mathematics with Applications, 55, 1629-1643(2008).

12. G. Gökdere and M. Gürcan, Laplace-Stieltjes transform of the system mean lifetime via geometric process model, Open Mathematics, 14, 384-392(2016). 